\title{
Development and characterization of polymorphic microsatellite loci
} for spiny-footed lizards, Acanthodactylus scutellatus group (Reptilia, Lacertidae) from arid regions

\author{
Sara Cristina Lopes ${ }^{1,2}$, Guillermo Velo-Antón ${ }^{1}$, Paulo Pereira', Susana Lopes ${ }^{1}$, Raquel Godinho ${ }^{1,2}$, \\ Pierre-André Crochet $^{3}$ and José Carlos Brito ${ }^{1,2^{*}}$
}

\begin{abstract}
Background: Spiny-footed lizards constitute a diverse but scarcely studied genus. Microsatellite markers would help increasing the knowledge about species boundaries, patterns of genetic diversity and structure, and gene flow dynamics. We developed a set of 22 polymorphic microsatellite loci for cross-species amplification in three taxa belonging to the Acanthodactylus scutellatus species group, A. aureus, A. dumerili/A. senegalensis and A. longipes, and tested the same markers in two other members of the group, A. scutellatus and A. taghitensis.

Results: Amplifications in A. aureus, A. longipes and A. dumerili/A. senegalensis were successful, with markers exhibiting a number of alleles varying between 1 and 19. Expected and observed heterozygosity ranged, respectively, between $0.046-0.893$ and 0.048-1.000. Moreover, 17 and 16 loci were successfully amplified in A. scutellatus and A. taghitensis, respectively.
\end{abstract}

Conclusion: These markers are provided as reliable genetic tools to use in future evolutionary, behavioural and conservation studies involving species from the A. scutellatus group.

Keywords: Cross-species amplification, Nuclear markers, Population genetics, Sahara-Sahel

\section{Background}

Spiny-footed lizards, or fringe-toed lizards (genus Acanthodactylus), form a clade of small ground-dwelling lizards occurring mostly in arid regions $[1,2]$. The genus is the most specious of the Lacertidae family and is widely distributed, occurring from the Iberian Peninsula, south of the Mediterranean Basin, across the Sahara-Sahel, Arabian Peninsula, and as far east as India [1, 2]. Being often abundant and occupying different types of open, flat habitats, these lizards are important elements of the

\footnotetext{
*Correspondence: jcbrito@cibio.up.pt

${ }^{2}$ Departamento de Biologia, Faculdade de Ciências, Universidade do

Porto. Rua Campo Alegre s/n, 4169-007 Porto, Portugal

Full list of author information is available at the end of the article
}

vertebrate communities of deserts and arid ecosystems in North Africa and Arabia. Despite their diversity, knowledge about most of the species is still scarce and their taxonomy is partly unresolved [1-4]. Most authors agree on splitting Acanthodactylus into several species groups or complexes [1,4]. The $A$. scutellatus species group shows one of most complex taxonomies [2, 5-8]. It includes six species according to the last global revision (A. aureus, $A$. dumerili, A. longipes, A. scutellatus, A. senegalensis and A. taghitensis) [3]. However, urgent systematic revision based on molecular data is needed given that: (1) eastern populations previously attributed to A. longipes are now considered a new species (A. aegyptius, [7]); and (2) species boundaries in $A$. scutellatus, $A$. longipes, $A$. dumerili and $A$. senegalensis as currently defined remain uncertain 
(own unpublished data, SC Lopes, Velo-Antón, Crochet, Brito). The species group has multiple forms occurring in sympatry in Mauritania-A. aureus, A. dumerili, A. senegalensis, and $A$. longipes [9]. In this contact zone, morphologically intermediate individuals were previously observed [3] and molecular studies are needed to distinguish whether high morphological diversity or hybridization explain these intermediate morphotypes. In addition, assessment of gene flow in such areas of sympatry would be critical for a better understanding of the species boundaries. Microsatellite markers have been extremely useful, and affordable, for addressing numerous topics in conservation and evolutionary biology, allowing, e.g., gene flow and population structure assessments, demographic inferences and genetic diversity estimation [10-12]. Yet, no microsatellite markers are available for the Acanthodactylus genus.

Here we describe a set of 22 polymorphic microsatellite loci (tri- and tetranucleotides) characterized in four species included in the $A$. scutellatus species group ( $A$. aureus, $A$. longipes and $A$. dumerili/A. senegalensis). Considering the uncertain species boundaries for $A$. dumer$i l i$ and $A$. senegalensis, we chose to refer to them as $A$. dumerili/A. senegalensis in the following sections. We further tested cross-amplification of these markers in two other members of the species group, A. scutellatus and $A$. taghitensis.

\section{Methods}

A genomic library was constructed from 12 specimens of A. aureus, collected across the species' distribution. A tissue sample was collected from the tail tip by following ethical guidelines for use of live reptiles (http:// www.aaalac.org/accreditation/Guidelines_for_Use_of_ Live_Amphibians_and_Reptiles.pdf). All specimens were released on site after sample collection. Fieldwork was developed with permission from the Ministére Délégué auprès du Premier Ministre Chargé de l'Environnement, Nouakchott (Permit: 460/MDE/PNBA) and from the Haut Commissariat aux Eaux et Forêts et à la Lutte Contre la Désertification, Rabat (Permits 2562012 and 20-2013). Analyses were done at a CITES registered laboratory: 13PT0065/S. Field collection and handling practices were approved by the Committee of Animal Experimentation of the University of Porto (Portugal) under the Directive 2010/63/EU of the European Parliament.

Genomic DNA extractions were performed from tissue samples using EasySpin Kit (Qiagen), following an adapted protocol for tissue samples (with minor adjustments to centrifugation and incubation conditions) and then pooled in equimolar concentrations. The changes to the extraction protocol were as follows: after adding the $A B$ solution, we centrifuged at $3700 \mathrm{rpm}$ for $4 \mathrm{~min}$ (instead of $4000 \mathrm{rpm}$ for $2 \mathrm{~min}$ ). After adding the Wash solution, we centrifuged at $3700 \mathrm{rpm}$ for $6 \mathrm{~min}$ (instead of $8000 \mathrm{rpm}$ for $1 \mathrm{~min}$ ). After repeating the Wash solution step and discarding flow-through, we centrifuged at $3700 \mathrm{rpm}$ for $10 \mathrm{~min}$ (instead of $14,000 \mathrm{rpm}$ for $5 \mathrm{~min}$ ). After adding the Elution Buffer, we incubated at $55^{\circ}$ for $15 \mathrm{~min}$ (instead of $50^{\circ}$ for $10 \mathrm{~min}$ ). Last centrifugation was at $3700 \mathrm{rpm}$ (instead of 14,000 rpm). Microsatellite isolation was developed through 454 GS-FLX Titanium pyrosequencing of enriched DNA libraries [13]. This process was developed by GenoScreen (http://www.pasteurlille.fr/fr/recherche/plateformes/tordeux_plat.html) and included sequence data quality control, assembly and analyses, and primer design. Initially, 50 loci were selected from the library and tested for amplification using seven samples of $A$. aureus, $A$. dumerili/A. senegalensis, and $A$. longipes. Thirty loci amplified reliably, producing fragments of the expected size. Twenty-two were polymorphic (Table 1), and amplified with differential success in the following target species: 21 in A. aureus, 18 in A. longipes and 15 in A. dumerili/A. senegalensis. These 22 loci were therefore used for genotyping 38 samples of $A$. aureus, 35 of $A$. longipes, and 43 of $A$. dumerili/A. senegalensis, collected along coastal Morocco and Mauritania (Table 2; Fig. 1). Markers were multiplexed in four reactions, using M13-primer genotyping protocol with four different dye-labelled tails, and forward primer concentration of 1/10 of dye-labelled reverse primer [14] (Table 1). The transferability of the primers was tested by crossamplification of five specimens of $A$. scutellatus (from Morocco, Tunisia, Libya, Algeria and Egypt) and one specimen of $A$. taghitensis (Mauritania). PCR amplifications were conducted using the Multiplex PCR Kit (QIAGEN) following manufacturer's instructions in a final $10 \mu \mathrm{l}$ volume, always in the presence of a negative control. Touchdown PCR conditions started with an initial denaturation step of $15 \mathrm{~min}$ at $95{ }^{\circ} \mathrm{C}$; first round (nine cycles) of $30 \mathrm{~s}$ at $95{ }^{\circ} \mathrm{C}, 90 \mathrm{~s}$ for annealing (decreasing $0.5{ }^{\circ} \mathrm{C}$ per cycle) at $58-54{ }^{\circ} \mathrm{C}$ (Multiplexes 1, 2 and 3) or $55-51{ }^{\circ} \mathrm{C}$ (Multiplex 4), and $30 \mathrm{~s}$ at $72{ }^{\circ} \mathrm{C}$; second round ( 31 cycles) of $30 \mathrm{~s}$ at $95{ }^{\circ} \mathrm{C}, 1 \mathrm{~min}$ at $54{ }^{\circ} \mathrm{C}$ (Multiplexes 1, 2 and 3), or $51{ }^{\circ} \mathrm{C}$ (Multiplex 4), $30 \mathrm{~s}$ at $72{ }^{\circ} \mathrm{C}$, and a final extension of $30 \mathrm{~min}$ at $60{ }^{\circ} \mathrm{C}$. Amplification was performed in Biorad T100 Thermal Cyclers, and the PCR products were later separated by capillary electrophoresis on an automatic sequencer ABI3130xl Genetic Analyzer (AB Applied Biosystems). Fragments were scored against the GeneScan-500 LIZ Size Standard using the GENEMAPPER 4.1 (Applied Biosystems) and manually checked twice. Potential 
Table 1 Global characterization of the 22 microsatellite loci characterized in Acanthodactylus aureus, A. dumerili/A. senegalensis and $A$. longipes

\begin{tabular}{|c|c|c|c|c|c|c|}
\hline Locus & GenBank assess no. & Repeat & Primer sequence $\left(5^{\prime}-3^{\prime}\right)$ & Multiplex & TD & Dye \\
\hline $\mathrm{Ac1}$ & KU295182 & $(\mathrm{ATAC})_{8}$ & $\begin{array}{l}\text { F: CTGTGGTATATCCCCTGCCA } \\
\text { R: GGTGGCTTCTCCACAGCTATT }\end{array}$ & 1 & $58^{\circ} / 54^{\circ}$ & FAM \\
\hline Ac4 & KU295183 & $(\mathrm{TTC})_{21}$ & $\begin{array}{l}\text { F: ACAGCTCTGCAGTAATTCCATTT } \\
\text { R: CCGATGCAGTGTTTCGTAGG }\end{array}$ & 3 & $58^{\circ} / 54^{\circ}$ & VIC \\
\hline Ac5 & KU295184 & $(\mathrm{AAC})_{15}$ & $\begin{array}{l}\text { F: GTTGCTTCAACTGCTCCTCC } \\
\text { R: AGTGTCCTGTGCACAACCAG }\end{array}$ & 1 & $58^{\circ} / 54^{\circ}$ & VIC \\
\hline Ac6 & KU295185 & $(\mathrm{TTG})_{10}$ & $\begin{array}{l}\text { F: GTAGCCCAGTCAGATGGGTG } \\
\text { R: CCTCCAACATTCCAGTCCAG }\end{array}$ & 4 & $55^{\circ} / 51^{\circ}$ & NED \\
\hline Ac8 & KU295186 & $(\mathrm{TTG})_{11}$ & $\begin{array}{l}\text { F: GACATCTGAAGGCAGCCCTA } \\
\text { R: GGTTGTAGCCTGGAGCAGAA }\end{array}$ & 1 & $58^{\circ} / 54^{\circ}$ & NED \\
\hline Ac9 & KU295187 & $(\mathrm{CAA})_{15}$ & $\begin{array}{l}\text { F:TCATACAGGGATGTTTCAGGG } \\
\text { R: GCAGGAGGAAGGAAGCTTTTT }\end{array}$ & 1 & $58^{\circ} / 54^{\circ}$ & PET \\
\hline Ac13 & KU295188 & $(\mathrm{AAC})_{14}$ & $\begin{array}{l}\text { F: TCCATGGGGTCACAAAGAGT } \\
\text { R:TCTCCAGCACTTATCTGATGC }\end{array}$ & 2 & $58^{\circ} / 54^{\circ}$ & FAM \\
\hline Ac14 & KU295189 & $(\mathrm{CAA})_{10}$ & $\begin{array}{l}\text { F: TTAAGTGGCAATGTGTTGCAT } \\
\text { R:TCCCACATGGTGGGTTACTT }\end{array}$ & 2 & $58^{\circ} / 54^{\circ}$ & VIC \\
\hline Ac16 & KU295190 & $(A G G)_{10}$ & $\begin{array}{l}\text { F: AGTCAATTTATTCAAATGATCTTCCA } \\
\text { R: TCATCCAAGAAAATCTGCTGC }\end{array}$ & 2 & $58^{\circ} / 54^{\circ}$ & VIC \\
\hline Ac19 & KU295191 & $(\mathrm{AAC})_{14}$ & $\begin{array}{l}\text { F: TCATTTCACTTCAAACCTGTGG } \\
\text { R: ACTGATGTTGGGTTTGGAGC }\end{array}$ & 2 & $58^{\circ} / 54^{\circ}$ & PET \\
\hline Ac20 & KU295192 & $(\mathrm{GTT})_{11}$ & $\begin{array}{l}\text { F: ATGCATAAGTACGAAAAGGGGA } \\
\text { R: TCTACAGAGAAAGAGAAATAACAACAA }\end{array}$ & 2 & $58^{\circ} / 54^{\circ}$ & PET \\
\hline Ac23 & KU295193 & $(\mathrm{CAT})_{8}$ & $\begin{array}{l}\text { F: GCGAACATGCACAAGGTTT } \\
\text { R: ACCCTGCTTGGTTCTCATTG }\end{array}$ & 1 & $58^{\circ} / 54^{\circ}$ & FAM \\
\hline Ac28 & KU295194 & $(\mathrm{ACAT})_{8}$ & $\begin{array}{l}\text { F: TGTCCGAAATAGGATGGAGC } \\
\text { R: GGAAAGCCAATGCCTCTACA }\end{array}$ & 4 & $55^{\circ} / 51^{\circ}$ & PET \\
\hline Ac31 & KU295195 & $(\mathrm{GTT})_{10}$ & $\begin{array}{l}\text { F: GAAGGGTTACAACTGCCTGG } \\
\text { R:CAGTGCTTCAGCAACAGGAG }\end{array}$ & 4 & $55^{\circ} / 51^{\circ}$ & FAM \\
\hline Ac32 & KU295196 & $(\mathrm{TTC})_{15}$ & $\begin{array}{l}\text { F:TAGTCCGTAAACTTGTGGGTCA } \\
\text { R:TTCTCAGACAACAGACACCCA }\end{array}$ & 3 & $58^{\circ} / 54^{\circ}$ & FAM \\
\hline Ac33 & KU295197 & $(\mathrm{TGT})_{16}$ & $\begin{array}{l}\text { F: GGCACTGAAATATGTGGTTTTG } \\
\text { R:TGACATGCTTCGGTGAAGTC }\end{array}$ & 3 & $58^{\circ} / 54^{\circ}$ & FAM \\
\hline Ac36 & KU295198 & $(\mathrm{TGT})_{9}$ & $\begin{array}{l}\text { F: GTCACGTTGATTGCATTGCT } \\
\text { R: GCCAACTGGGAAACCTAGC }\end{array}$ & 3 & $58^{\circ} / 54^{\circ}$ & VIC \\
\hline Ac43 & KU295199 & $(\mathrm{CAA})_{13}$ & $\begin{array}{l}\text { F: AGCTTTTGTACGTTCCTTTGC } \\
\text { R: CCAGAGAAACACATATGCAAGC }\end{array}$ & 4 & $55^{\circ} / 51^{\circ}$ & FAM \\
\hline Ac44 & KU295200 & $(\mathrm{GGA})_{11}$ & $\begin{array}{l}\text { F:TCCTTAAGAAAGGTACTTAATGCCA } \\
\text { R:TCTTTACGTAGTCCCTTTGTGG }\end{array}$ & 4 & $55^{\circ} / 51^{\circ}$ & VIC \\
\hline Ac45 & KU295201 & $(\mathrm{CAA})_{10}$ & $\begin{array}{l}\text { F: AGGCAATGGAAGACAGGGA } \\
\text { R: GCCTACAGTTTGTGCATAGGG }\end{array}$ & 4 & $55^{\circ} / 51^{\circ}$ & VIC \\
\hline Ac47 & KU295202 & $(\mathrm{ACA})_{11}$ & $\begin{array}{l}\text { F: CTTGCCTCTTCGCTTTCTGT } \\
\text { R:TCCGGACAGCATTCCTCTAC }\end{array}$ & 4 & $55^{\circ} / 51^{\circ}$ & NED \\
\hline Ac49 & KU295203 & $(\mathrm{AAC})_{11}$ & $\begin{array}{l}\text { F: CAAAGAAAATTGTTGGAGGGG } \\
\text { R: GTAAAACATCGGAAGGCAGC }\end{array}$ & 4 & $55^{\circ} / 51^{\circ}$ & PET \\
\hline
\end{tabular}

$T D$ touchdown temperatures

evidences of null alleles, allelic dropouts and stuttering were assessed using MICRO-CHECKER v2.2.3 [15] at each locus, for each population. Tests for HardyWeinberg equilibrium (HWE) and linkage disequilibrium (LD) were assessed in GENEPOP online version (http://wbiomed.curtin.edu.au/genepop/); with subsequent Bonferroni correction in both cases. Observed and expected heterozygosity were computed using GenAlEx v6.501 [16]. For some populations, samples were obtained from different localities. Consequently, analyses were based on groups of samples that are not necessarily panmitic populations, which probably accounts for deviations from Hardy-Weinberg equilibrium. 
Table 2 Data on sampling localities for each species

\begin{tabular}{|c|c|c|c|c|c|}
\hline Code & Species & Latitude & Longitude & Local & Country \\
\hline 6477 & A. aureus & 20.9444 & -16.5494 & Kerekchet et Teintâne, extreme N & Mauritania \\
\hline A366 & A. aureus & 21.2182 & -16.8432 & Nouâdhibou, 40 km S of & Mauritania \\
\hline A367 & A. aureus & 21.2182 & -16.8432 & Nouâdhibou, 40 km S of & Mauritania \\
\hline A368 & A. aureus & 21.2182 & -16.8432 & Nouâdhibou, 40 km S of & Mauritania \\
\hline A369 & A. aureus & 21.2182 & -16.8432 & Nouâdhibou, 40 km S of & Mauritania \\
\hline A358 & A. aureus & 21.0978 & -16.6998 & Nouâdhibou, 70 km S of & Mauritania \\
\hline A359 & A. aureus & 21.0978 & -16.6998 & Nouâdhibou, $70 \mathrm{~km} \mathrm{~S} \mathrm{of}$ & Mauritania \\
\hline A360 & A. aureus & 21.0978 & -16.6998 & Nouâdhibou, 70 km S of & Mauritania \\
\hline A361 & A. aureus & 21.0978 & -16.6998 & Nouâdhibou, $70 \mathrm{~km} \mathrm{~S} \mathrm{of}$ & Mauritania \\
\hline A362 & A. aureus & 21.0978 & -16.6998 & Nouâdhibou, 70 km S of & Mauritania \\
\hline A363 & A. aureus & 21.0978 & -16.6998 & Nouâdhibou, 70 km S of & Mauritania \\
\hline 6449 & A. aureus & 20.8233 & -16.5882 & PNBA: Kerekchet et Teintâne, central & Mauritania \\
\hline 6458 & A. aureus & 20.8023 & -16.5718 & PNBA: Kerekchet et Teintâne, central & Mauritania \\
\hline 5171 & A. aureus & 20.7190 & -16.6195 & PNBA: Kerekchet et Teintâne, central 2 & Mauritania \\
\hline 5172 & A. aureus & 20.7190 & -16.6195 & PNBA: Kerekchet et Teintâne, central 2 & Mauritania \\
\hline 5173 & A. aureus & 20.7251 & -16.6291 & PNBA: Kerekchet et Teintâne, W side 1 & Mauritania \\
\hline 5176 & A. aureus & 20.7620 & -16.6183 & PNBA: Kerekchet et Teintâne, W side 3 & Mauritania \\
\hline 6443 & A. aureus & 20.7764 & -16.6287 & PNBA: Kerekchet et Teintâne, Western face & Mauritania \\
\hline 6446 & A. aureus & 20.8115 & -16.6158 & PNBA: Kerekchet et Teintâne, Western face & Mauritania \\
\hline 6448 & A. aureus & 20.8115 & -16.6158 & PNBA: Kerekchet et Teintâne, Western face & Mauritania \\
\hline 6435 & A. aureus & 20.7938 & -16.5462 & PNBA: Sebkhet Dbâdeb et Teintâne, W margin & Mauritania \\
\hline A437 & A. aureus & 28.8731 & -10.7027 & Aoreora, 15 km E of (Plage Blanche) & Morocco \\
\hline A438 & A. aureus & 28.8731 & -10.7027 & Aoreora, 15 km E of (Plage Blanche) & Morocco \\
\hline A439 & A. aureus & 28.8731 & -10.7027 & Aoreora, 15 km E of (Plage Blanche) & Morocco \\
\hline A440 & A. aureus & 28.8731 & -10.7027 & Aoreora, 15 km E of (Plage Blanche) & Morocco \\
\hline A441 & A. aureus & 28.8731 & -10.7027 & Aoreora, 15 km E of (Plage Blanche) & Morocco \\
\hline A442 & A. aureus & 28.8731 & -10.7027 & Aoreora, 15 km E of (Plage Blanche) & Morocco \\
\hline A443 & A. aureus & 28.8731 & -10.7027 & Aoreora, 15 km E of (Plage Blanche) & Morocco \\
\hline A435 & A. aureus & 28.7447 & -10.7438 & Aoreora, $25 \mathrm{~km} \mathrm{~S} \mathrm{of}$ & Morocco \\
\hline A436 & A. aureus & 28.7447 & -10.7438 & Aoreora, $25 \mathrm{~km} \mathrm{~S} \mathrm{of}$ & Morocco \\
\hline A556 & A. aureus & 29.8511 & -9.7706 & Bou Soun & Morocco \\
\hline 10,625 & A. aureus & 28.5177 & -11.2970 & Douira, N of & Morocco \\
\hline 10,638 & A. aureus & 28.3701 & -11.4387 & Douira, $\mathrm{S}$ of & Morocco \\
\hline 10,634 & A. aureus & 28.1544 & -11.9117 & Laareig & Morocco \\
\hline 10,636 & A. aureus & 27.9291 & -12.2945 & Leirane & Morocco \\
\hline 9048 & A. aureus & 28.9662 & -10.6000 & Plage Blanche & Morocco \\
\hline 10,635 & A. aureus & 28.0875 & -12.0814 & Sidi Akhfennir & Morocco \\
\hline 10,624 & A. aureus & 28.5479 & -10.9583 & Tafnidilt & Morocco \\
\hline 6470 & A. dum./sen. & 20.9172 & -16.5418 & Kerekchet et Teintâne, extreme N & Mauritania \\
\hline 6473 & A. dum./sen. & 20.9204 & -16.5415 & Kerekchet et Teintâne, extreme N & Mauritania \\
\hline 6474 & A. dum./sen. & 20.9204 & -16.5415 & Kerekchet et Teintâne, extreme N & Mauritania \\
\hline 3618 & A. dum./sen. & 20.0500 & -16.0582 & PNBA: Adeim el Marrâr & Mauritania \\
\hline 5111 & A. dum./sen. & 19.9733 & -16.1874 & PNBA: Agreigrât, 1 km E of & Mauritania \\
\hline 6384 & A. dum./sen. & 20.1010 & -16.1655 & PNBA: Aguilâl & Mauritania \\
\hline 5126 & A. dum./sen. & 20.1287 & -16.1581 & PNBA: Aguilâl 1 & Mauritania \\
\hline 5135 & A. dum./sen. & 20.1497 & -16.1420 & PNBA: Aguilâl 4 & Mauritania \\
\hline 5120 & A. dum./sen. & 20.1498 & -16.1719 & PNBA: Aguilâl, 1 km W of & Mauritania \\
\hline 5158 & A. dum./sen. & 20.7802 & -16.3944 & PNBA: Amgheououas es Sâhli & Mauritania \\
\hline 5160 & A. dum./sen. & 20.7843 & -16.4027 & PNBA: Amgheououas es Sâhli & Mauritania \\
\hline
\end{tabular}


Table 2 continued

\begin{tabular}{|c|c|c|c|c|c|}
\hline Code & Species & Latitude & Longitude & Local & Country \\
\hline 5162 & A. dum./sen. & 20.8007 & -16.4227 & PNBA: Amgheououas es Sâhli, 3 km NW of & Mauritania \\
\hline 6390 & A. dum./sen. & 20.1808 & -16.1474 & PNBA: Dlo'Matai & Mauritania \\
\hline 6391 & A. dum./sen. & 20.1808 & -16.1474 & PNBA: Dlo'Matai & Mauritania \\
\hline 6394 & A. dum./sen. & 20.2330 & -16.1247 & PNBA: Dlo'Matai & Mauritania \\
\hline 2750 & A. dum./sen. & 20.2789 & -16.1003 & PNBA: Dló Matai & Mauritania \\
\hline 3622 & A. dum./sen. & 20.0934 & -16.0613 & PNBA: Grâret Zra & Mauritania \\
\hline 2768 & A. dum./sen. & 20.8070 & -16.5701 & PNBA: Kerekchet et Teintâne & Mauritania \\
\hline 2769 & A. dum./sen. & 20.8070 & -16.5701 & PNBA: Kerekchet et Teintâne & Mauritania \\
\hline 6450 & A. dum./sen. & 20.8233 & -16.5882 & PNBA: Kerekchet et Teintâne, central & Mauritania \\
\hline 6453 & A. dum./sen. & 20.8233 & -16.5882 & PNBA: Kerekchet et Teintâne, central & Mauritania \\
\hline 6456 & A. dum./sen. & 20.8023 & -16.5718 & PNBA: Kerekchet et Teintâne, central & Mauritania \\
\hline 6457 & A. dum./sen. & 20.8023 & -16.5718 & PNBA: Kerekchet et Teintâne, central & Mauritania \\
\hline 6460 & A. dum./sen. & 20.8283 & -16.5672 & PNBA: Kerekchet et Teintâne, central & Mauritania \\
\hline 6461 & A. dum./sen. & 20.8283 & -16.5672 & PNBA: Kerekchet et Teintâne, central & Mauritania \\
\hline 6462 & A. dum./sen. & 20.8283 & -16.5672 & PNBA: Kerekchet et Teintâne, central & Mauritania \\
\hline 6463 & A. dum./sen. & 20.8283 & -16.5672 & PNBA: Kerekchet et Teintâne, central & Mauritania \\
\hline 6468 & A. dum./sen. & 20.8294 & -16.5518 & PNBA: Kerekchet et Teintâne, central & Mauritania \\
\hline 6469 & A. dum./sen. & 20.8294 & -16.5518 & PNBA: Kerekchet et Teintâne, central & Mauritania \\
\hline 5181 & A. dum./sen. & 20.7831 & -16.5865 & PNBA: Kerekchet et Teintâne, central 3 & Mauritania \\
\hline 6445 & A. dum./sen. & 20.8115 & -16.6158 & PNBA: Kerekchet et Teintâne, Western face & Mauritania \\
\hline 2763 & A. dum./sen. & 20.8060 & -16.4561 & PNBA: N of Baie d'Arguin & Mauritania \\
\hline 2743 & A. dum./sen. & 20.0964 & -16.1798 & PNBA: NE of El Mounâne & Mauritania \\
\hline 6377 & A. dum./sen. & 20.1233 & -16.1266 & PNBA: Oued Nouafferd & Mauritania \\
\hline 5139 & A. dum./sen. & 20.1574 & -16.1037 & PNBA: Oued Nouafferd 3 & Mauritania \\
\hline 6375 & A. dum./sen. & 20.0845 & -16.1313 & PNBA: Oued Nouafferd, $2 \mathrm{~km} \mathrm{~S} \mathrm{of}$ & Mauritania \\
\hline 6376 & A. dum./sen. & 20.0845 & -16.1313 & PNBA: Oued Nouafferd, $2 \mathrm{~km} \mathrm{~S} \mathrm{of}$ & Mauritania \\
\hline 3615 & A. dum./sen. & 20.0928 & -16.1059 & PNBA: Râs Tafarît, 16 km E of & Mauritania \\
\hline 6433 & A. dum./sen. & 20.8173 & -16.4858 & PNBA: Sebkhet Dbâdeb et Teintâne, 2 km E of & Mauritania \\
\hline 6431 & A. dum./sen. & 20.7791 & -16.4602 & PNBA: Sebkhet Dbâdeb et Teintâne, 4 km E of & Mauritania \\
\hline 6426 & A. dum./sen. & 20.7395 & -16.4150 & PNBA: Sebkhet Dbâdeb et Teintâne, 8 km SE of & Mauritania \\
\hline 6363 & A. dum./sen. & 19.9808 & -16.1016 & PNBA: Taguîlâlet Jreik, 2 km W of & Mauritania \\
\hline 6364 & A. dum./sen. & 19.9808 & -16.1016 & PNBA: Taguîlâlet Jreik, 2 km W of & Mauritania \\
\hline 2745 & A. longipes & 20.0699 & -16.0896 & PNBA: 5 km E of El Mounâne & Mauritania \\
\hline 6319 & A. longipes & 19.6589 & -16.2639 & PNBA: Ackenjeîl & Mauritania \\
\hline 6320 & A. longipes & 19.6589 & -16.2639 & PNBA: Ackenjeîl & Mauritania \\
\hline 6369 & A. longipes & 20.0567 & -16.0993 & PNBA: Adeim el Marrâr, 4 km W of & Mauritania \\
\hline 6383 & A. longipes & 20.1010 & -16.1655 & PNBA: Aguilâl & Mauritania \\
\hline 6386 & A. longipes & 20.1010 & -16.1655 & PNBA: Aguilâl & Mauritania \\
\hline 5119 & A. longipes & 20.1498 & -16.1719 & PNBA: Aguilâl, 1 km W of & Mauritania \\
\hline A344 & A. longipes & 20.5080 & -16.2380 & PNBA: Bir el Gareb, 15 km S of & Mauritania \\
\hline A345 & A. longipes & 20.5080 & -16.2380 & PNBA: Bir el Gareb, 15 km S of & Mauritania \\
\hline A346 & A. longipes & 20.5080 & -16.2380 & PNBA: Bir el Gareb, 15 km S of & Mauritania \\
\hline A347 & A. longipes & 20.5080 & -16.2380 & PNBA: Bir el Gareb, 15 km S of & Mauritania \\
\hline 6339 & A. longipes & 19.8079 & -16.1479 & PNBA: Elb en Nouçç, extreme $S$ & Mauritania \\
\hline 6340 & A. longipes & 19.8079 & -16.1479 & PNBA: Elb en Nouçç, extreme $S$ & Mauritania \\
\hline 6348 & A. longipes & 19.7819 & -16.1880 & PNBA: Grâret Agoueifa & Mauritania \\
\hline 6349 & A. longipes & 19.7819 & -16.1880 & PNBA: Grâret Agoueifa & Mauritania \\
\hline 6414 & A. longipes & 20.5046 & -16.3389 & PNBA: Îmgoûtene, 5 km NE of & Mauritania \\
\hline 3607 & A. longipes & 19.8232 & -16.2100 & PNBA: louîk, 16 km SE of & Mauritania \\
\hline
\end{tabular}


Table 2 continued

\begin{tabular}{|c|c|c|c|c|c|}
\hline Code & Species & Latitude & Longitude & Local & Country \\
\hline 6451 & A. longipes & 20.8233 & -16.5882 & PNBA: Kerekchet et Teintâne, central & Mauritania \\
\hline 6452 & A. longipes & 20.8233 & -16.5882 & PNBA: Kerekchet et Teintâne, central & Mauritania \\
\hline 5168 & A. longipes & 20.7328 & -16.6021 & PNBA: Kerekchet et Teintâne, central 1 & Mauritania \\
\hline 5163 & A. longipes & 20.7538 & -16.5820 & PNBA: Kerekchet et Teintâne, E side 1 & Mauritania \\
\hline 5164 & A. longipes & 20.7538 & -16.5820 & PNBA: Kerekchet et Teintâne, E side 1 & Mauritania \\
\hline 5167 & A. longipes & 20.7309 & -16.5902 & PNBA: Kerekchet et Teintâne, E side 2 & Mauritania \\
\hline 6438 & A. longipes & 20.6815 & -16.5913 & PNBA: Kerekchet et Teintâne, extreme S & Mauritania \\
\hline 5177 & A. longipes & 20.7620 & -16.6183 & PNBA: Kerekchet et Teintâne, W side 3 & Mauritania \\
\hline 6317 & A. longipes & 19.6522 & -16.2803 & PNBA: Kôra & Mauritania \\
\hline 6318 & A. longipes & 19.6522 & -16.2803 & PNBA: Kôra & Mauritania \\
\hline 2746 & A. longipes & 20.1281 & -16.0893 & PNBA: Oued Nouafferd & Mauritania \\
\hline 5137 & A. longipes & 20.1507 & -16.1211 & PNBA: Oued Nouafferd 1 & Mauritania \\
\hline 6374 & A. longipes & 20.0845 & -16.1313 & PNBA: Oued Nouafferd, 2 km S of & Mauritania \\
\hline 6436 & A. longipes & 20.7938 & -16.5462 & PNBA: Sebkhet Dbâdeb et Teintâne, W margin & Mauritania \\
\hline 6352 & A. longipes & 19.7942 & -16.2101 & PNBA: Taguîlâlet Jreik & Mauritania \\
\hline 6356 & A. longipes & 19.8455 & -16.2014 & PNBA: Taguîlâlet Jreik, 1 km W of & Mauritania \\
\hline 6302 & A. longipes & 19.5863 & -16.3268 & PNBA: Toueigueret, 1 km SW of & Mauritania \\
\hline 6306 & A. longipes & 19.5842 & -16.3514 & PNBA: Toueigueret, 2 km SW of & Mauritania \\
\hline A768 & A. scutellatus & 33.5833 & 2.9500 & Bou Trekfine & Algeria \\
\hline A787 & A. scutellatus & 22.7666 & 25.6000 & Gilf Kebir & Egypt \\
\hline A133 & A. scutellatus & 32.8968 & 12.1536 & Jadi Resort; 7 km E of Zuara & Libya \\
\hline 8992 & A. scutellatus & 32.3665 & -1.3191 & Oued Es Safsaf, dunes above dam & Morocco \\
\hline A086 & A. scutellatus & 33.9000 & 8.0489 & Tozeur, 7 km W of & Tunisia \\
\hline 5823 & A.taghitensis & 22.8047 & -12.3783 & Zouérat, 11 km NE of & Mauritania \\
\hline
\end{tabular}

Coordinates are in decimal degrees (WGS84 projection)

PNBA Parc National du Banc d'Arguin

\section{Results and discussion}

MICRO-CHECKER revealed no evidence of allelic dropout or stuttering, and no heterozygote excess was observed. In addition, no loci appeared to be in linkage disequilibrium. Table 3 summarizes occurrence of heterozygote deficiency and suspected null alleles for all loci in all populations in the three target species. While the occurrence of null alleles would limit the use of some of these markers in the affected species, other departures from Hardy-Weinberg equilibrium probably result from pooling several sampling localities in the same "populations" (see above). Additionally, even markers showing such evidences might be adequate to apply in other populations and they are applicable in at least one of these species.

All loci genotyped for each species were polymorphic (Table 4), except for Ac44 that amplified only for A. longipes. The Ac36 was also monomorphic in
A. dumerili/A. senegalensis tested populations but polymorphism was observed in inland samples of this species (own unpublished data, Lopes, Velo-Antón, Crochet, Brito). The number of alleles per locus varied between 5 and 19 in A. aureus, and between 1 and 9 in $A$. dumerili/A. senegalensis and A. longipes. Expected and observed heterozygosity varied, respectively, between $0.594-0.893 / 0.188-1.000$ in A. aureus, $0.223-0.829 / 0.154-0.826$ in $A$. dumerili/A. senegalensis (ignoring Ac36), and 0.046-0.862/0.048-0.905 in A. longipes (ignoring Ac44). Most markers amplified in both $A$. scutellatus/17 loci) and A. taghitensis (16 loci).

Although the applicability of each marker may depend on the species considered, the information provided in our work allows a selection of good markers for future use on assessments of genetic structure, genetic diversity, gene flow, and demographic inferences, expanding the 


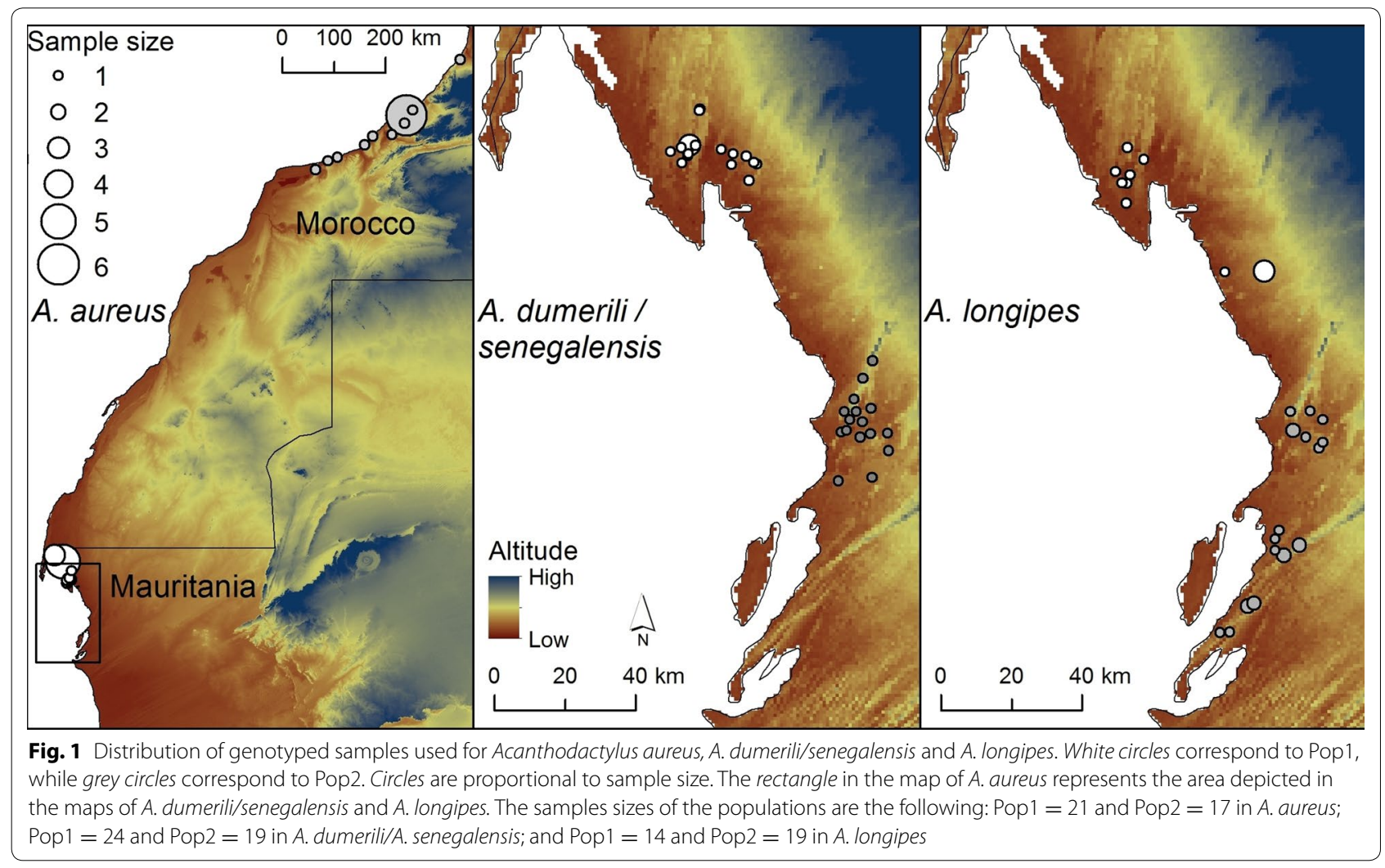

Table 3 Observations of heterozygote deficiency and null alleles

\begin{tabular}{|c|c|c|c|c|c|c|c|c|c|c|c|c|}
\hline & \multicolumn{4}{|l|}{ A. aureus } & \multicolumn{4}{|c|}{ A. longipes } & \multicolumn{4}{|c|}{ A. dumerili/senegalensis } \\
\hline & \multicolumn{2}{|l|}{ Pop1 } & \multicolumn{2}{|l|}{ Pop2 } & \multicolumn{2}{|l|}{ Pop1 } & \multicolumn{2}{|l|}{ Pop2 } & \multicolumn{2}{|l|}{ Pop1 } & \multicolumn{2}{|l|}{ Pop2 } \\
\hline & Het. Def. & Null alleles & Het. Def. & Null alleles & Het. Def. & Null alleles & Het. Def. & Null alleles & Het. Def. & Null alleles & Het. Def. & Null alleles \\
\hline Ac4 & & & & & & $*$ & & $*$ & - & - & - & - \\
\hline Ac5 & & & & & & & & & * & * & & \\
\hline Ac6 & * & * & * & * & - & - & - & - & * & * & * & * \\
\hline Ac13 & & & & & & & & & & * & & * \\
\hline Ac16 & $*$ & $*$ & & * & & & & & - & - & - & - \\
\hline Ac19 & & & & & & & * & * & & & & \\
\hline Ac23 & & & & & & & & * & & & & \\
\hline Ac31 & & & & & & & * & * & & $*$ & & * \\
\hline Ac32 & & & & & & & & & & $*$ & & $*$ \\
\hline Ac33 & * & * & * & * & & & & & $*$ & $*$ & & \\
\hline Ac43 & & & & & & & & & $*$ & * & & * \\
\hline Ac45 & & & * & * & & & & & & & & \\
\hline
\end{tabular}

Results are presented for Acanthodactylus aureus, A. dumerili/senegalensis and A. longipes. Significant values after Bonferroni correction are marked with an asterisk. Since the heterozygote deficiency was estimated in GENEPOP while null alleles were assessed in MICROCHECKER, differences in the estimation methods may explain the observed lack of concordance between heterozygote deficiency and null alleles in some cases

- , markers that failed to amplify in a certain species 


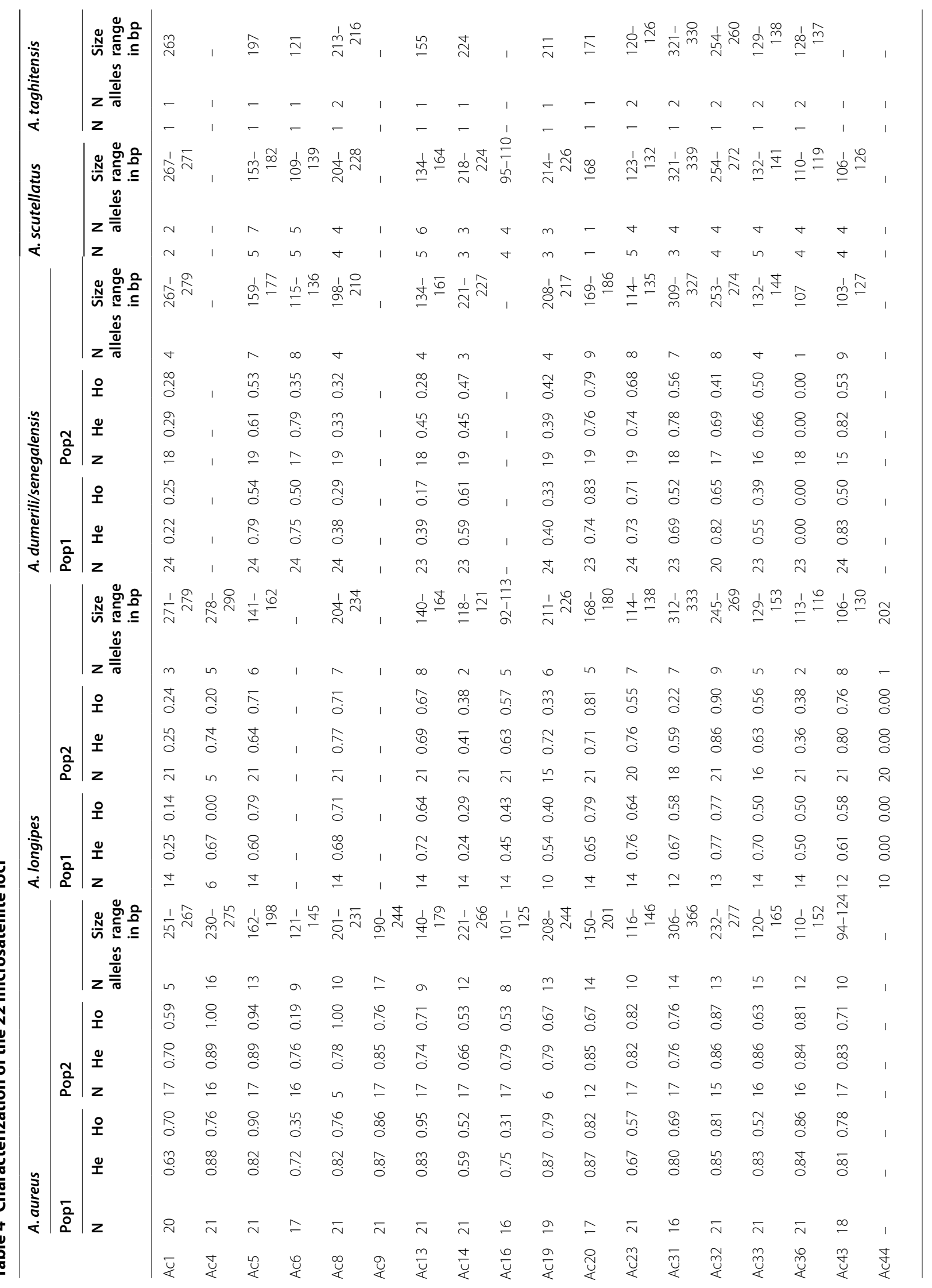




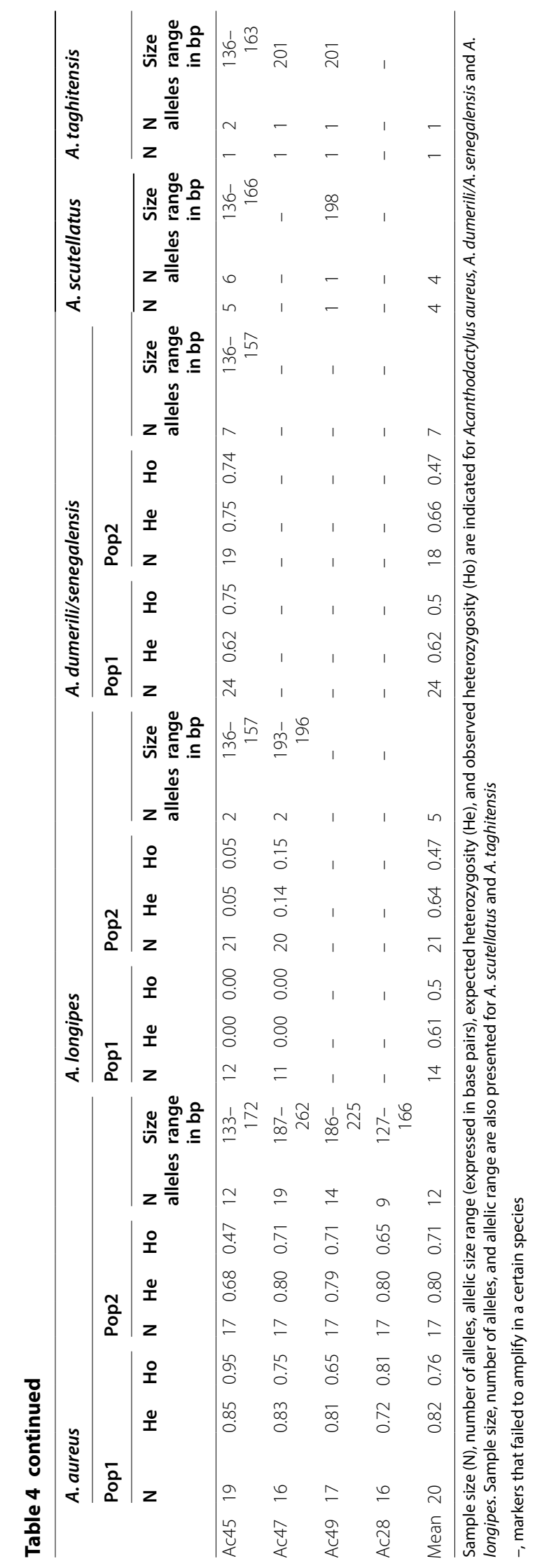


possible themes for evolutionary, behavioural and conservation studies in this species group.

\section{Authors' contributions}

$\mathrm{SCL}$ carried out the laboratory tasks, performed the molecular analyses, and drafted the manuscript. PP and SL participated in the microsatellite marker optimization and validation. GVA, PAC and RG contributed to the molecular analyses. JCB designed and supervised the study. All authors read and approved the final manuscript.

\section{Author details}

${ }^{1} \mathrm{ClBIO} / \mathrm{InBIO}$, Centro de Investigação em Biodiversidade e Recursos Genéticos, Universidade do Porto. Campus Agrário de Vairão, 4485-661 Vairão, Portugal. ${ }^{2}$ Departamento de Biologia, Faculdade de Ciências, Universidade do Porto. Rua Campo Alegre s/n, 4169-007 Porto, Portugal. ${ }^{3}$ CNRS-UMR 5175, Centre d'Ecologie Fonctionnelle et Evolutive, 1919 route de Mende, 34293 Montpellier-Cedex 5, France.

\section{Acknowledgements}

This study was partially supported by Mohammed bin Zayed Species Conservation Fund (project 11052709), by National Geographic Society (Grants CRE 7629-04 and 8412-08), by FEDER funds through the Operational Programme for Competitiveness Factors-COMPETE (FCOMP-01-0124-FEDER-028276), and by National Funds through FCT, Foundation for Science and Technology (PTDC/BIA-BIC/2903/2012). Research conducted in the scope of the LIA known as "Biodiversity and Evolution". GVA, RG and JCB are supported by FCT contracts (IF/01425/2014, IF/00564/2012, and IF/00459/2013, respectively).

\section{Competing interests}

The authors declare that they have no competing interests.

Received: 1 July 2015 Accepted: 30 November 2015

Published online: 17 December 2015

\section{References}

1. Salvador A. A revision of the lizards of the genus Acanthodactylus (Sauria: Lacertidae). Bon Zool Monog. 1982;16:1-167.

2. Arnold EN. Osteology, genitalia and the relationships of Acanthodactylus (Reptilia: Lacertidae). Bull Brit Mus Nat Hist (Zoology). 1983;44:291-339.

3. Crochet PA, Geniez P, Ineich I. A multivariate analysis of the fringe-toed lizards of the Acanthodactylus scutellatus group (Squamata: Lacertidae): systematic and biogeographical implications. Zool J Linnean Soc. 2003;137:117-55.
4. Harris D, Arnold E. Elucidation of the relationships of spiny-footed lizards, Acanthodactylus spp. (Reptilia: Lacertidae) using mitochondrial DNA sequence, with comments on their biogeography and evolution. J Zool. 2000;252:351-62

5. Bons J. Les lacertiliens du Sud-Ouest Marocain: systématique, répartition géographique, éthologie, écologie). Trav Inst Sci Chérifien. 1959;18:1-130.

6. Mellado J, Olmedo G. El género Acanthodactylus en Marruecos: problemas de identificación en los grupos de especies A. pardalis y A. scutellatus. Amphib Reptilia. 1990;11:131-46.

7. Baha El Din SM. A contribution to the herpetology of Sinai. Brit Herp Soc Bul. 1994;48:18-27.

8. Baha El Din SM. A new lizard of the Acanthodactylus scutellatus group (Squamata: Lacertidae) from Egypt. Zool Middle East. 2007:40:21-32.

9. Sindaco R, Jeremcenko VK. The reptiles of the Western Palearctic: annotated checklist and distributional atlas of the turtles, crocodiles, amphisbaenians and lizards of Europe, North Africa, Middle East and Central Asia. Latina: Edizioni Belvedere, Monografie della Societas Herpetologica Italica; 2008.

10. Schlotterer $C$. The evolution of molecular markers: just a matter of fashion? Nat Rev Genet. 2004;5:63-9.

11. Wan $\mathrm{QH}$, Wu H, Fujihara T, Fang SG. Which genetic marker for which conservation genetics issue? Electrophoresis. 2004;25:2165-76.

12. Selkoe KA, Toonen RJ. Microsatellites for ecologists: a practical guide to using and evaluating microsatellite markers. Ecol Lett. 2006;9:615-29.

13. Malausa T, Gilles A, Meglecz E, Blanquart H, Duthoy S, Costedoat C, et al. High-throughput microsatellite isolation through 454 GS-FLX titanium pyrosequencing of enriched DNA libraries. Mol Ecol Res. 2011;11:638-44.

14. Schuelke M. An economic method for the fluorescent labelling of PCR fragments. Nat Biotech. 2000;18:233-4.

15. Van Oosterhout C, Hutchinson WF, Wills DP, Shipley P. MICRO-CHECKER: software for identifying and correcting genotyping errors in microsatellite data. Mol Ecol Notes. 2004;4:535-8.

16. Peakall R, Smouse PE. GenAIEx 6.5: genetic analysis in Excel. Population genetic software for teaching and research-an update. Bioinformatics. 2012;28:2537-9.

\section{Submit your next manuscript to BioMed Central and we will help you at every step:}

- We accept pre-submission inquiries

- Our selector tool helps you to find the most relevant journal

- We provide round the clock customer support

- Convenient online submission

- Thorough peer review

- Inclusion in PubMed and all major indexing services

- Maximum visibility for your research

Submit your manuscript at www.biomedcentral.com/submit

\section{() Biomed Central}

